\title{
Prophylactic Embolization of the Cystic Artery Before Radioembolization: Feasibility, Safety, and Outcomes
}

\author{
Justin P. McWilliams $\cdot$ Stephen T. Kee • \\ Christopher T. Loh $\cdot$ Edward W. Lee $\cdot$ \\ David M. Liu
}

Received: 27 June 2010/ Accepted: 7 October 2010/Published online: 11 November 2010

(C) The Author(s) 2010. This article is published with open access at Springerlink.com

\begin{abstract}
Purpose To evaluate the safety and efficacy of two different methods of proximal cystic artery embolization in patients undergoing yttrium-90 radioembolization.

Materials and Methods Forty-six patients had cystic artery embolization performed immediately before yttrium90 radioembolization, either by using Gelfoam pledgets $(n=35)$ or coils $(n=11)$. Clinical symptomatology during the admission and angiographic findings at 1-month follow-up were retrospectively reviewed. Rates of collateralization or recanalization of the cystic artery were compared, as well as the frequency of postprocedural abdominal pain and need for cholecystectomy.

Results Technical success was achieved in all patients, and there were no procedural complications related to cystic artery embolization. Of the 11 coil-embolized patients, $5(45 \%)$ demonstrated collateralization of the
\end{abstract}

The material was presented as a scientific exhibit at CIRSE 2009.

J. P. McWilliams · S. T. Kee · C. T. Loh ·

E. W. Lee - D. M. Liu

Department of Interventional Radiology, David Geffen School

of Medicine at UCLA, Los Angeles, CA, USA

J. P. McWilliams ( $\square)$

757 Westwood Plaza, 2nd floor, Suite 2125C,

Los Angeles, CA 90095, USA

e-mail: jumcwilliams@mednet.ucla.edu

D. M. Liu

Department of Radiology, Angio-Interventional Section, Faculty of Medicine, University of British Columbia, Vancouver, BC V5Z1M9, Canada

D. M. Liu

Vancouver General Hospital, JP Pavilion G873,

855 W. 12th Avenue, Vancouver, BC V5Z1M9, Canada cystic artery at 1 month, and 1 (9\%) demonstrated recanalization of the cystic artery. Of the 35 Gelfoam-embolized cases, $2(6 \%)$ had collateralized at 1 month, and $14(40 \%)$ had recanalized. Two patients (one from each group) had self-limited right upper quadrant pain after the procedure, and one patient in the coil embolization group required cholecystectomy.

Conclusion Proximal cystic artery embolization is safe and feasible and may be performed during liver-directed embolotherapy to minimize the exposure of the gallbladder to particulate, chemoembolic, or radioembolic agents.

Keywords Cystic $\cdot$ Embolization $\cdot$ Yttrium ·

Radioembolization

\section{Introduction}

Yttrium-90 radioembolization is an effective technique for treatment of primary and secondary liver tumors [1-8]. A feared complication of the procedure is nontarget embolization of the radiopharmaceutical, which can result in severe damage to the stomach, duodenum, or pancreas. Nontarget embolization to the gastrointestinal tract is avoided by preprocedure coil embolization of the relevant splanchnic arteries [9-11].

Nontarget embolization of the gallbladder is also a concern during hepatic embolotherapy. Chemical or ischemic cholecystitis may result from transarterial chemoembolization or transarterial embolization, with occasional clinical sequelae including cholecystitis, cholecystectomy, and a trend to more severe postembolization syndrome [12-16]. Similarly, radiation cholecystitis may occur after radioembolization, and though it is usually subclinical, studies suggest it contributes to postprocedure morbidity, 
and occasionally necessitates cholecystectomy [5-8, 1719].

During radioembolization, gallbladder protection is usually afforded by lobar infusion distal to the cystic artery origin. However, a high incidence of cystic artery variation has been observed [20-22] which may complicate or preclude distal infusion. Also, infusion too distal in the lobar artery may prevent symmetric distribution of the radiopharmaceutical [23]. Prophylactic embolization of the cystic artery may be desirable in order to optimize the point of radiopharmaceutical administration or simplify dose planning.

In this study, gallbladder protection was attempted by using either Gelfoam or coil embolization of the cystic artery immediately before radioembolization. Periprocedural and postprocedural clinical course of the patients, as well as angiographic appearance of the embolized cystic artery at 1-month follow-up, were examined.

\section{Materials and Methods}

\section{Patient Characteristics}

Forty-six consecutive patients (31 male, 15 female; age range $27-72$ years, median 47 years) admitted for sequential lobar yttrium-90 radioembolization over a 1-year period at a single institution were retrospectively reviewed. Twenty-seven patients had hepatocellular carcinoma, and 19 patients suffered from metastatic liver disease, primarily from colorectal carcinoma. Institutional review board approval for this retrospective analysis was granted.

\section{Preprocedure}

Informed consent was obtained, which included mesenteric angiography, embolization, and radioembolization procedures. All patients underwent standard workup and evaluation, including history, physical, and relevant laboratory tests. A dedicated triple-phase computed tomographic scan was performed to map the relevant arterial anatomy and aid in treatment planning.

\section{Mesenteric Angiography}

Mesenteric angiography and vascular optimization was performed per institutional protocol [9]. Moderate sedation was induced, and the common femoral artery was accessed. Superselective angiography of the right and left hepatic arteries was performed, with injection rates that allowed for reflux into the proximal order vessel. Vascular optimization of the gastroduodenal artery, right gastric artery, falciform artery, gastrohepatic trunk, accessory left hepatic artery, supraduodenal artery, and replaced right hepatic artery was performed as necessary, utilizing coils and/or Gelfoam pledgets. Technetium- $99 \mathrm{~m}$ macroaggregated albumin $\left.{ }^{99 \mathrm{~m}} \mathrm{Tc}-\mathrm{MAA}\right)$ scanning via planar single-photon emission computed tomography (SPECT) was performed to detect any unobserved splanchnic flow and to estimate pulmonary shunt. At the time of mesenteric angiography, the cystic artery was identified, but not embolized.

\section{Cystic Artery Embolization and Right Hepatic Artery Radioembolization}

Seven to 21 days after the mesenteric mapping, the patients returned for right lobe radioembolization (all patients underwent sequential lobar administration). At this time, the cystic artery, and accessory vessels, when present, were superselected with a microcatheter (Renegade Hi-Flo, Boston Scientific, Natick, MA or Progreat Omega, Terumo, Somerset, NJ), and embolization of the proximal cystic artery was performed to stasis with either absorbable gelatin sponge pledgets (Gelfoam, Pfizer, New York, NY) $(n=35)$ or fibered microcoils (VortX-18, Boston Scientific, Natick, MA) $(n=11)$, at the operator's discretion. Gelfoam embolization technique involved cutting a small pledget of noncompressed Gelfoam of approximately $3 \mathrm{~mm}$ length and $1.5 \mathrm{~mm}$ width, rolling it between the fingers and backloading it into a $1 \mathrm{cc}$ syringe of contrast. The syringe was wet-mounted to the microcatheter, which had been preflushed with saline, and the pledget was injected with moderate force under fluoroscopic guidance. With either embolization technique, a subsequent postembolization angiogram was performed immediately proximal to the cystic artery to confirm lack of opacification of the gallbladder. Selective radioembolization of the right hepatic artery then proceeded routinely.

\section{Postprocedure Care and Clinical Follow-up}

Routine postprocedure care was provided, including overnight admission with close observation and pain and nausea control. The presence of right upper quadrant pain and positive Murphy's sign was recorded, if present, and presence of these symptoms prompted observation and surgical consultation. The need for cholecystectomy was dictated by progressive symptoms and discretion of the surgeon. Upon discharge, patients were instructed to contact the office for increasing pain, nausea, or fever.

Angiographic Follow-up

Approximately 1 month after the cystic artery embolization and right hepatic radioembolization procedure, 
patients returned for radioembolization of the left hepatic artery. This procedure commenced with proper hepatic angiography, with attention to the status of the previously embolized cystic artery. The status of gallbladder supply was described as persistent occlusion, if no flow to the gallbladder was seen; recanalization, if the embolized cystic artery had reconstituted; or collateralization, if new arterial feeders to the gallbladder had developed.

\section{Statistical Analysis}

The status of gallbladder supply at follow-up was classified as either a favorable outcome (persistent occlusion or recanalization) or an adverse outcome (collateralization or cholecystectomy) for each patient. The outcomes of the coil embolization group were then compared to the outcomes of the Gelfoam embolization group by Fisher's exact test. The level of significance used was $P<0.05$.

\section{Results}

Technical success, defined as complete embolization of the cystic artery immediately before right hepatic radioembolization, was achieved in all patients. No cystic artery perforations were noted.

\section{Clinical Consequence}

Two patients in the coil embolization group suffered right upper quadrant abdominal pain and positive Murphy's sign after the procedure. One patient was successfully treated conservatively, while the second patient had progressive symptoms and required cholecystectomy.

One patient in the Gelfoam embolization group suffered right upper quadrant abdominal pain and positive Murphy's sign after the procedure. This patient was managed conservatively with eventual resolution of symptoms. No patients in the Gelfoam embolization group required operative intervention.

\section{Fate of the Embolized Cystic Artery}

The status of the embolized cystic artery at 1-month angiographic follow-up is summarized in Fig. 1. In the coil embolization group, 1 patient $(9 \%)$ required cholecystectomy before angiographic follow-up. Four (36\%) of the 11 patients demonstrated persistent absence of flow to the gallbladder at 1-month follow-up, and 1 patient (9\%) demonstrated recanalization of the coil-embolized cystic artery. Five patients (45\%) demonstrated interval development of small collateral vessels supplying the gallbladder (Fig. 2).

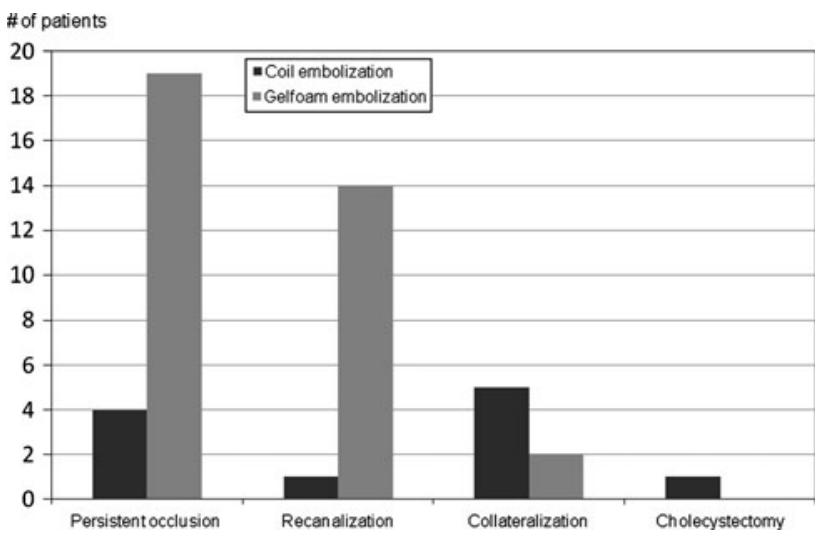

Fig. 1 Fate of the cystic artery at 1-month follow-up

In the Gelfoam embolization group, 19 (54\%) of 35 patients demonstrated persistent absence of flow to the gallbladder at 1-month follow-up. Two patients (6\%) demonstrated collateralization of flow. Fourteen patients (40\%) demonstrated interval recanalization (Fig. 3).

Patients treated with Gelfoam embolization were more likely to have a favorable outcome (persistent occlusion or recanalization) at angiographic follow-up compared to coil-embolized patients ( $94 \%$ vs. $45 \%, P=0.001)$.

\section{Conclusions}

Nontarget embolization of the gallbladder has been a frequent topic of discussion since the advent of hepatic arterial embolization procedures as a result of its proximity to the hepatic arterial supply. Gallbladder infarction is common when the cystic artery is occluded during chemoembolization, and the severity of infarction may correlate with the severity of postprocedural abdominal pain [14, 15]. With improvements in microcatheter technology and the trend toward superselective embolization, gallbladder infarction from lobar or whole liver transarterial chemoembolization became less relevant.

The development of yttrium-90 radioembolization, in which lobar administration of the radiopharmaceutical is standard protocol, has again brought nontarget embolization to the forefront of clinical concern. The gastroduodenal artery and right gastric artery are often protectively coil-embolized before radioembolization; this approach has proven quite safe thanks to the rich collateral networks in these vascular beds. Oversight or lack of embolic protection manifests as gastric or duodenal ulcers, which can be severe $[11,18]$. The cystic artery, however, is an end artery and has not been routinely protected before radioembolization as a result of concerns of gallbladder infarction. Not surprisingly, imaging findings of radiation cholecystitis are detected in up to $24 \%$ of patients after right lobe radioembolization [5]. Almost all of these patients can be 
Fig. 2 A Initial common hepatic angiogram demonstrates conventional origin of the cystic artery (arrow) and its characteristic forked appearance. B Selective cystic artery angiogram demonstrates accessory supply to hepatic parenchyma (open arrow) as well as a branch supplying the abdominal wall (closed arrow). C Immediately after coil embolization of the cystic artery (arrow), no persistent flow is present. Radioembolization was performed safely. D Follow-up proper hepatic angiography performed 1 month later demonstrates collateralization to the coil-embolized cystic artery (arrow)

Fig. 3 A Initial right hepatic angiogram demonstrates origin of the cystic artery (arrow) just before the division of the right hepatic artery into anterior and posterior sectoral branches.

B Selective angiography confirms gallbladder supply. C Immediately after Gelfoam embolization of the cystic artery, there is cessation of flow to the gallbladder (arrow). Radioembolization was performed safely. D Proper hepatic angiography 1 month later demonstrates recanalization of the cystic artery (arrow) without collateralization
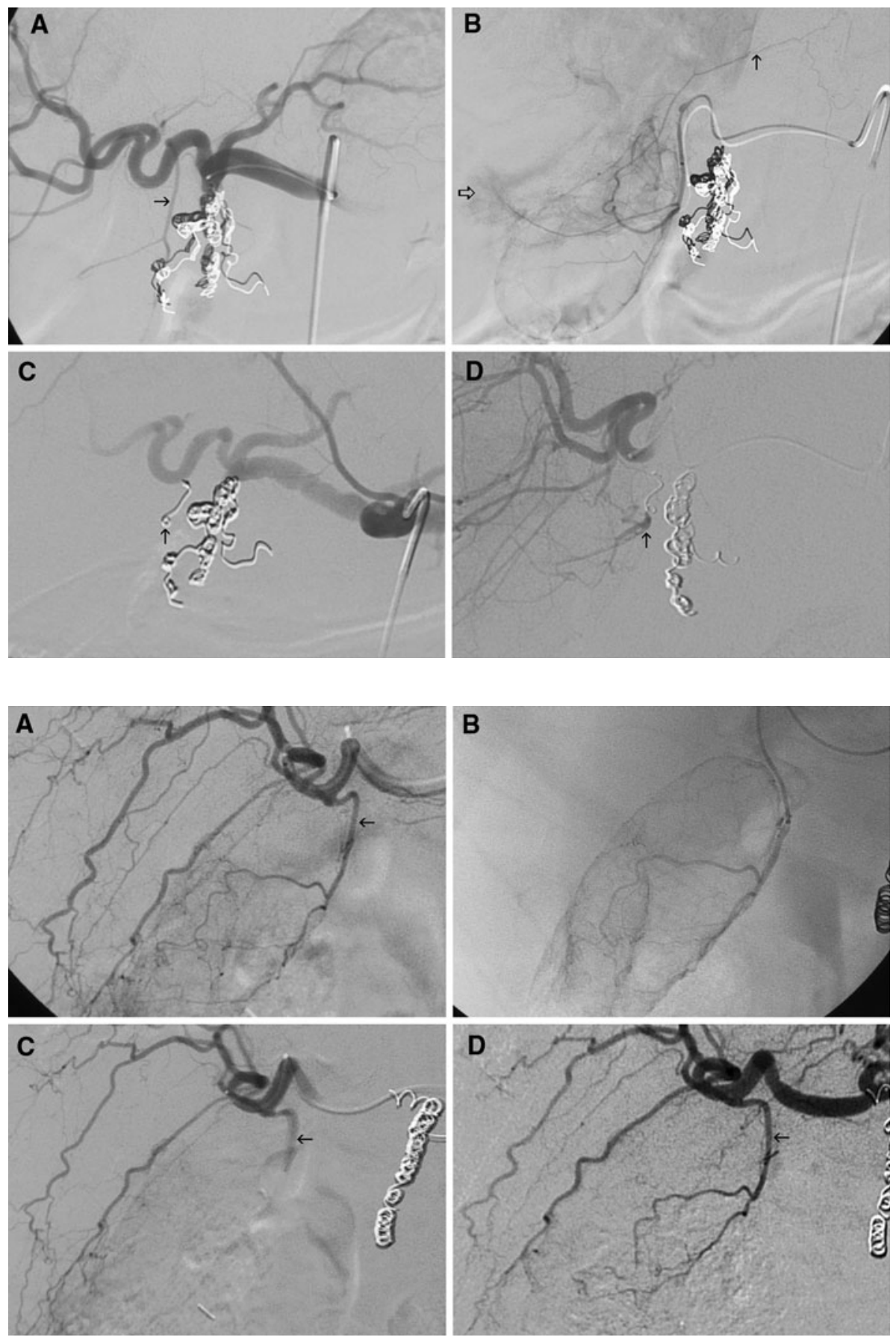

managed conservatively, but severe toxicity requiring cholecystectomy does occasionally occur.

Given the potential of gallbladder-related complications, protective embolization of the cystic artery may be relevant in circumstances where the location of the cystic artery would result in direct injection of therapeutic or significantly complicate dose planning. Prophylactic embolization of the cystic artery has been previously reported, particularly when the cystic artery demonstrates significant flow, or if catheterization distal to the cystic artery would lead to inadequate distribution of microspheres [23]. A few case reports in patients with pseudoaneurysms of the cystic artery have suggested that proximal coil embolization of the cystic artery can be performed without clinical sequelae [24, 25]. Surgical and radiologic reports suggest that tiny collaterals in the gallbladder fossa often communicate between the cystic and hepatic arterial circulations [26-28]. Such vessels would effectively render a dual blood supply to the gallbladder, increasing its tolerance to proximal embolization (Fig. 4). 
Fig. 4 A Hepatic angiography demonstrates cystic artery origin (arrow) from the left hepatic artery. B Selective angiography of the cystic artery demonstrates gallbladder supply (closed arrows) as well as multiple communications with the hepatic arterial circulation (open arrows)
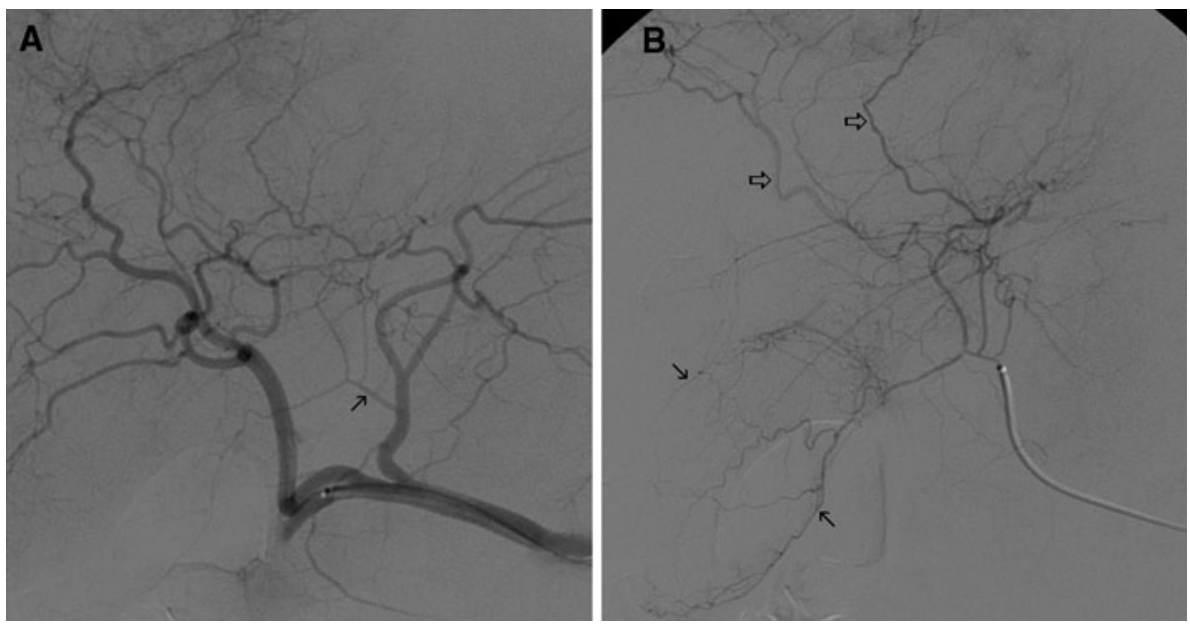

The cystic artery is classically identified by its Y-shaped configuration, corresponding to superficial and deep branches, and by its expected distribution in the gallbladder fossa. Surgical series demonstrate that the cystic artery arises conventionally (from the main trunk of the right hepatic artery) in only approximately half of patients. The cystic artery often arises more distally in the right hepatic artery, originating from the anterior sectoral artery in $16-23 \%$ of cases, the posterior sectoral artery in $4-5 \%$ of cases, and occasionally from segmental branches [21, 29]. These variants would be expected to be at higher risk during radioembolization, as right lobar infusion, even with catheterization deep into the right hepatic artery, would place the gallbladder in the embolization zone (Fig. 5). Origin from a replaced or accessory right hepatic artery, or origin from the left hepatic artery, may also affect radioembolization and should be recognized. Some less common variants, such as proper hepatic, common hepatic, gastroduodenal, or superior mesenteric artery supply, would place the cystic artery proximal to the infusion site and would likely increase the safety margin for radioembolization.

In this study, we found that protective cystic artery embolization before yttrium-90 administration is technically feasible. Despite prior reports of the technical difficulty of cystic artery embolization with Gelfoam [23] and occasional reports of cystic artery perforation [30], embolization was technically achievable in all of our patients without perforation. Furthermore, cystic artery embolization has an acceptable safety profile, with only three patients reporting clinical symptoms suggestive of cholecystitis, and one patient (in the coil embolization group) requiring cholecystectomy.

The ideal embolic agent for protective embolization of the cystic artery would provide effective proximal occlusion of the cystic artery without deep tissue penetration which could induce necrosis. Logical choices include coils and

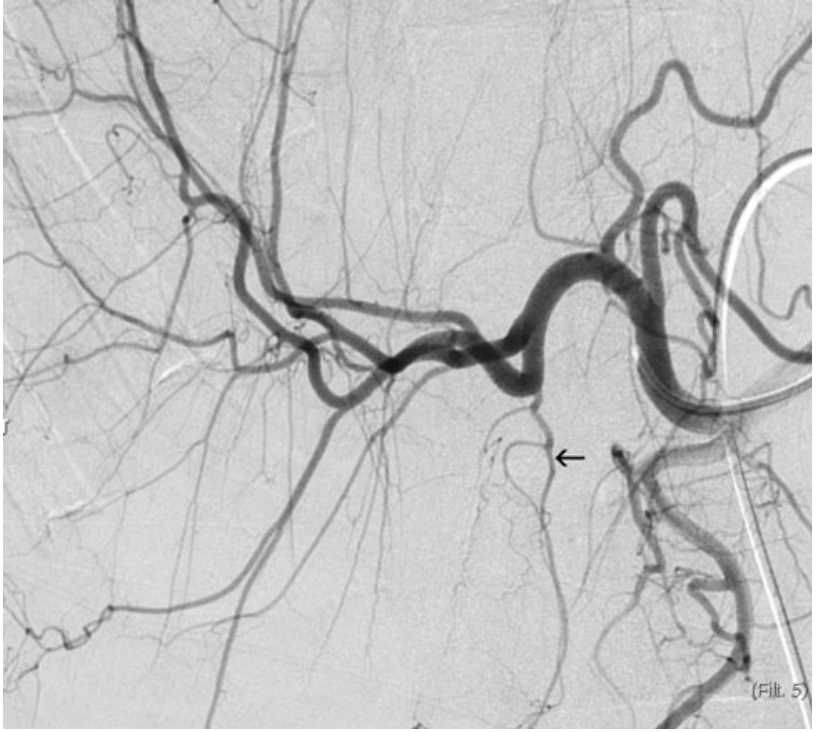

Fig. 5 Cystic artery origin (arrow) from the anterior sectoral branch of the right hepatic artery. This variant, occurring in approximately $20 \%$ of individuals, places the gallbladder in the treatment zone for right lobar radioembolization

Gelfoam pledgets. Furthermore, the embolic agent should not result in collateralization of flow to the embolized gallbladder, as small collateral vessels would be difficult or impossible to protect should repeat radioembolization prove necessary. Persistent occlusion of the cystic artery or recanalization without collateral formation would each be acceptable late outcomes. Our study suggests that Gelfoam pledgets are the embolic agent of choice, as they provided effective proximal occlusion with a low rate of collateralization, and without any instances of operative cholecystitis.

The findings of our study may also have implications for other embolization procedures in the hepatic vasculature. With the increased potency of carrier-based embolotherapeutics (such as drug eluting microspheres), minimizing nontarget embolization has become increasingly essential. 
In patients where subselective administration of the embolic agent is not possible or not desired (e.g. widespread disease requiring lobar infusion), or in cases where an aberrant cystic artery origin places the gallbladder in the target zone for embolization, prophylactic embolization of the cystic artery may allow more central administration of the embolic agent to be performed safely.

In our study, no gallbladder activity was detected on the planar SPECT scans performed after 99mTc-MAA administration, and results of the SPECT study did not influence cystic artery embolization. The use of SPECTcomputed tomography rather than planar SPECT improves detection of tracer deposition in the gallbladder [31] and might therefore serve as a marker for increased risk of radiation cholecystitis. This warrants further study, as it may allow selectivity of patients who could most benefit from prophylactic cystic artery embolization.

Limitations of this study include its relatively small size, particularly in the coil embolization group, and its retrospective nature without case control. Given the historically low rates of operative cholecystitis reported in the literature without cystic artery protection, it is not clear if the rate of cholecystitis would have been different if cystic artery embolization had not been performed. Also, the clinical symptoms of the patients could be confounded, as the patients underwent cystic artery embolization and hepatic radioembolization concurrently. Finally, angiographic follow-up was only obtained at 1 month; it is uncertain whether more collateralization of flow to the gallbladder would have occurred had more time been allowed to elapse.

In conclusion, proximal cystic artery embolization is safe and feasible and may be performed during liverdirected embolotherapy to minimize the exposure of the gallbladder to particulate, chemoembolic, or radioembolic agents. In our study, Gelfoam pledgets seem to be the embolic of choice, providing adequate embolization with delayed recanalization and limited collateral formation.

Conflict of interest D.M.L. is a consultant for Sirtex Medical and Biosphere Medical. The other authors declare that they have no conflict of interest.

Open Access This article is distributed under the terms of the Creative Commons Attribution Noncommercial License which permits any noncommercial use, distribution, and reproduction in any medium, provided the original author(s) and source are credited.

\section{References}

1. Andrews JC, Walker SC, Ackerman RJ et al (1994) Hepatic radioembolization with yttrium-90 containing glass microspheres: preliminary results and clinical follow-up. J Nucl Med 35: $1637-1644$
2. Herba MJ, Illescas FF, Thirlwell MP et al (1988) Hepatic malignancies: improved treatment with intraarterial Y-90. Radiology 169:311-314

3. Herba MJ, Thirlwell MP (2002) Radioembolization for hepatic metastases. Semin Oncol 29:152-159

4. Lewandowski RJ, Thurston KG, Goin JE et al (2005) ${ }^{90} \mathrm{Y}$ microsphere (TheraSphere) treatment for unresectable colorectal cancer metastases of the liver: response to treatment at targeted doses of $135-150 \mathrm{~Gy}$ as measured by $\left[{ }^{18} \mathrm{~F}\right]$ fluorodeoxyglucose positron emission tomography and computed tomographic imaging. J Vasc Interv Radiol 16:1641-1651

5. Miller FH, Keppke AL, Reddy D et al (2007) Response of liver metastases after treatment with yttrium-90 microspheres: role of size, necrosis, and PET. Am J Roentgenol 188:776-783

6. Sato KT, Lewandowski RJ, Mulcahy MF et al (2008) Unresectable chemorefractory liver metastases: radioembolization with Y-90 microspheres-safety, efficacy, and survival. Radiology 247: $507-515$

7. Jakobs TF, Hoffmann RT, Dehm K et al (2008) Hepatic yttrium90 radioembolization of chemotherapy-refractory colorectal cancer liver metastases. J Vasc Interv Radiol 19:1187-1195

8. Carr BI (2004) Hepatic arterial yttrium-90 glass microspheres (Therasphere) for unresectable hepatocellular carcinoma: interim safety and survival data on 65 patients. Liver Transplantation 10(2(suppl 1)):S107-S110

9. Liu DM, Salem R, Bui JT et al (2005) Angiographic considerations in patients undergoing liver-directed therapy. J Vasc Interv Radiol 16:911-935

10. Lewandowski RJ, Sato KT, Atassi B et al (2007) Radioembolization with Y-90 microspheres: angiographic and technical considerations. Cardiovasc Intervent Radiol 30:571-592

11. Murthy R, Brown DB, Salem R et al (2007) Gastrointestinal complications associated with hepatic arterial Yttrium-90 microsphere therapy. J Vasc Interv Radiol 18:553-562

12. Tarazov PG, Polysalov VN, Prozorovskij KV et al (2000) Ischemic complications of transcatheter arterial chemoembolization in liver malignancies. Acta Radiol 41:156-160

13. Gates J, Hartnell GG, Stuart KE et al (1999) Chemoembolization of hepatic neoplasms: safety, complications, and when to worry. Radiographics 19:399-414

14. Leung DA, Goin JE, Sickles C et al (2001) Determinants of postembolization syndrome after hepatic chemoembolization. J Vasc Interv Radiol 12:321-326

15. Kuroda C, Iwasaki M, Tanaka T et al (1983) Gallbladder infarction following hepatic transcatheter arterial embolization. Radiology 149:85-89

16. Takayasu K, Moriyama N, Muramatsu Y et al (1985) Gallbladder infarction after hepatic artery embolization. Am J Roentgenol 144:135-138

17. Lewandowski RJ, Salem R (2004) Incidence of radiation cholecystitis in patients receiving Y-90 treatment for unresectable liver malignancies (abstract). J Vasc Interv Radiol 15:S162

18. Atassi B, Bangash AK, Lewandowski RJ et al (2008) Biliary sequelae following radioembolization with yttrium-90 microspheres. J Vasc Interv Radiol 19:691-697

19. Murthy R, Nunez R, Szklaruk J et al (2005) Yttrium-90 microsphere therapy for hepatic malignancy: devices, indications, technical considerations, and potential complications. Radiographics 25(suppl 1):S41-S55

20. Molmenti EP, Pinto PA, Klein J et al (2003) Normal and variant arterial supply of the liver and gallbladder. Pediatr Transpl 7:80-82

21. Mlakar B, Gadzijev EM, Ravnik D et al (2003) Anatomical variations of the cystic artery. Eur J Morphol 41:31-34

22. Price P, Holden C (1993) Anatomic variance in the cholecystic blood supply: a case report. Am Surg 59:278-280 
23. Salem R, Thurston KG (2006) Radioembolization with yttrium90 microspheres: a state-of-the-art brachytherapy treatment for primary and secondary liver malignancies. Part 2: special topics. J Vasc Interv Radiol 17:1425-1439

24. Bergey E, Einstein DM, Herts BR (1995) Cystic artery pseudoaneurysm as a complication of laparoscopic cholecystectomy. Abdom Imaging 20:75-77

25. Maeda A, Kunou T, Saeki S et al (2002) Pseudoaneurysm of the cystic artery with hemobilia treated by arterial embolization and elective cholecystectomy. J Hepatobiliary Pancreat Surg 9: 755-758

26. Polyzonis MB, Tsikaras P, Hytiroglou P (1989) Further observations on the vascular system of the gallbladder in man. Bull Assoc Anat 73:25-28

27. Michels NA (1966) Newer anatomy of the liver and its variant blood supply and collateral circulation. Am J Surg 112:337-347
28. Komatsu T, Matsui O, Kadoya M et al (1999) Cystic artery origin of the segment V hepatic artery. Cardiovasc Intervent Radiol 22: $165-167$

29. Couinaud C (1954) Distribution de l'artere hepatique dans le foie. Acta Anat 22:49-81

30. Miyayama S, Matsui O, Nishida H et al (2003) Transcatheter arterial chemoembolization for unresectable hepatocellular carcinoma fed by the cystic artery. J Vasc Interv Radiol 14(9 (pt1)): $1155-1161$

31. Ahmadzadehfar H, Sabet A, Biermann K et al (2010) The significance of ${ }^{99 \mathrm{~m}} \mathrm{Tc}-\mathrm{MAA}$ SPECT/CT liver perfusion imaging in treatment planning for ${ }^{90} \mathrm{Y}$-microsphere selective internal radiation treatment. J Nucl Med 51:1206-1212 